\title{
Ueber die Addition von Natriumacetessig- und Natriummalonsäureäthern zu den Aethern nngesättigter Säuren.
}

\author{
Zweite Mittheilung; \\ von \\ Arthur Michael und Paul C. Freer.
}

Das mir soeben zukommende Heft der Berichte (17, 307) enthält eine Mittheilung von K. Auwers, welche eine Fortsetzung der Versuche von dem einen von uns ${ }^{1}$ ) über die Addition von Natriumacetessig- und Natriummalonsäureäthern zu den Aethern ungesättigter Säuren ist und uns zur Veröffentlichung unserer Versuche über die Einwirkung von Zimmtsäureäther auf Natriumacetessigäther veranlasst. Es wurde früher nachgewiesen, dass diese Reaction einen abnormen Verlauf hat, indem aus dem zuerst entstehenden Additionsprodukt sogleich Natriumäthylat sich abspaltet, und obwohl wir nun eine grosse Anzahl von Versuchen über dieses Condensationsprodukt angestellt haben, ist es uns bis jetzt nicht gelungen, seine Constitution mit Sicherheit zu ermitteln.

Die von dem einen von uns schon beschriebene Verbindung $\mathrm{C}_{15} \mathrm{H}_{16} \mathrm{O}_{4}$ ist nicht das erste Produkt der Reaction. Dieses entsteht, wenn man Natriumacetessigäther und Zimmtsäureäther in äquivalenten Verhältnissen in alkoholischer Lösung etwa $z$ wölf Stunden bei $10^{\circ}$ sich überlässt, wobei grosse, durchsichtige Krystalle sich abscheiden, die man aus wenig warmem Alkohol umkrystallisiren kann. Dieser Körper besteht aus einem Additionsprodukt von der Zusammensetzung $\mathrm{C}_{6} \mathrm{H}_{5}-\mathrm{CH}-\mathrm{CH}-\mathrm{COOC}_{2} \mathrm{H}_{5}, 2 \mathrm{CH}_{3}-\mathrm{CONa}-\mathrm{CH}-\mathrm{COOC}_{2} \mathrm{H}_{5}$, und wird beim Stehen mit Wasser quantitativ in Zimmtsäureund Acetessigäther zersetzt. Bei diesem Versuch kommt demnach nur die Hälfte des angewandten Zimmtsäureäthers zur Wirkung, und in der That kann man aus der Mutterlauge von dem Additionsprodukt geradezu die Hälfte des genommenen Zimmtsäureäthers wieder gewinnen.

1) A. Michael, dies. Journ. [2] 35, 349. 
Natriumacetessig- u. Natriummalonsäureäthern etc. 391

Wird das natriumhaltige Additionsprodukt mit Alkohol erhitzt, so geht es leicht in das Condensationsprodukt $\mathrm{C}_{15} \mathrm{H}_{18} \mathrm{O}_{4}$ über, und zwar bilden sich $205 \mathrm{Grm}$. von dem Körper aus $242 \mathrm{Grm}$. Zimmtsäureäther. $\mathrm{Er}$ schmilzt bei $144^{\circ}-145^{0}$ ) und eine neue Analyse gab Zahlen, die sehr genau mit denen von der früher angegebenen Zusammensetzung $\mathrm{C}_{15} \mathrm{H}_{16} \mathrm{O}_{4}$ verlangten übereinstimmten.

Versetzt man diese Lösung von $\mathrm{C}_{15} \mathrm{H}_{16} \mathrm{O}_{4}$ in wässrigem Natriumcarbonat mit einer Lösung von Phenylhydrazinchlorhydrat und essigsaurem Natrium, so bildet sich das Hydrazon als amorpher, weisser Körper, welcher bei $74^{\circ}-7^{\circ}$ schmilzt, und dem die Zusammensetzung $\mathrm{C}_{15} \mathrm{H}_{16} \mathrm{O}_{3}-\mathrm{N}-\mathrm{NHC}_{6} \mathrm{H}_{6}$ zukommt.

Durch Verseifung von $\mathrm{C}_{15} \mathbf{H}_{18} \mathrm{O}_{4}$ mit alkoholischem Kali entsteht das Kaliumsalz einer Säure, welche aus der wässrigen Lösung durch Mineralsäuren gefällt wird und bei $95^{\circ}$ unter Abgabe von Kohlendioxyd schmilzt, um sogleich bei dieser Temperatur zu erstarren und nun erst bei $175^{\circ}$ wieder zu schmelzen. Die höher schmelzende Substanz hat die Zusammensetzung $\mathrm{C}_{12} \mathrm{H}_{12} \mathrm{O}_{2}$ und bildet lange, glänzende Nadeln, die bei $184^{\circ}-185^{\circ}$ schmelzen und in Alkohol und Aether leicht löslich, in Ligroïn unlöslich sind; sie löst sich ferner in wässrigem Natriumearbonat unter Entwicklung von Kohlendioxyd. Die Entstehung dieser Körper wird durch die folgenden Gleichungen dargestellt:

$$
\begin{aligned}
\mathrm{C}_{12} \mathrm{H}_{11} \mathrm{O}_{2} \mathrm{COOC}_{2} \mathrm{H}_{5}+\mathrm{H}_{2} \mathrm{O} & =\mathrm{C}_{12} \mathrm{H}_{11} \mathrm{O}_{2} \mathrm{COOH}+\mathrm{C}_{2} \mathrm{H}_{5} \mathrm{OH} \\
\mathrm{C}_{12} \mathrm{H}_{12} \mathrm{O}_{2} \mathrm{COOH} & =\mathrm{C}_{12} \mathrm{H}_{12} \mathrm{O}_{2}+\mathrm{CO}_{2} .
\end{aligned}
$$

Um zu ermitteln, ob die Verbindung $\mathrm{C}_{12} \mathrm{H}_{12} \mathrm{O}_{2}$ ein Carbonyl enthält, wurde sie in alkoholischer Lösung mit dem Phenylhydrazingemisch versetzt, wobei sogleich ein dunkles Oel sich abschied; und weder dieses, noch das feste Hydrazon, welches man erhielt, wenn eine Natriumcarbonatlösung von $\mathrm{C}_{12} \mathrm{H}_{12} \mathrm{O}_{2}$ auf ähnliche Weise behandelt wurde, konnten in reinem $\mathrm{Zu}$ stand erhalten werden. Ein besseres Resultat erhielten wir bei der Einwirkung von Hydroxylamin auf $\mathrm{C}_{12} \mathrm{H}_{12} \mathrm{O}_{2}$. Versetzt man eine neutrale Lösung des Körpers in Ammoniak mit einer wässrigen Lösung von Hydroxylaminchlorhydrat, so entsteht ein

1) Der Schmelzpunkt wurde früher als $140,5^{\circ}$ angegeben. 
weisser, amorpher Niederschlag, welcher in heissem Wasser sich auflöst und beim Erkalten der Lösung als ein weisses Pulver sich absetzt. Dieses Oxim schmilzt bei $129^{\circ}-131^{\circ}$ unter Zersetzung, und entsteht durch Condensation von zwei Molekülen $\mathrm{C}_{12} \mathrm{H}_{12} \mathrm{O}_{2}$ mit einem Molekül Hydroxylamin:

$$
2 \mathrm{C}_{1}, \mathrm{H}_{1}, \mathrm{O}_{2}+\mathrm{NH}_{2} \mathrm{OH}=\mathrm{C}_{24} \mathrm{H}_{22} \mathrm{O}_{3} \mathrm{~N}(\mathrm{OH})+\mathrm{H}_{2} \mathrm{O} \text {. }
$$

Ein zweites Oxim erhält man durch Einwirkung von freiem Hydroxylamin in wässriger Lösung auf eine alkoholische Lösung ron $\mathrm{C}_{12} \mathrm{H}_{12} \mathrm{O}_{2}$; es ist ebenfalls eine weisse, amorphe Substanz, die bei $172^{\circ}$ schmilzt und in Alkohol und Aether leicht, in Ligrö̈n unlöslich ist. Die Analysen dieses Oxims lieferten Zahlen, die der Zusammensetzung $\mathrm{C}_{13} \mathrm{H}_{12} \mathrm{ON}(\mathrm{OH})$ annähernd entsprachen, und dies berechtigt wohl zu dem Schluss, dass der Körper $\mathrm{C}_{12} \mathrm{H}_{12} \mathrm{O}_{2}$ ein Carbonyl enthält.

Erhitzt man äquivalente Mengen von Anilin und $\mathrm{C}_{12} \mathrm{H}_{12} \mathrm{O}_{2}$ in alkoholischer Lösung, so scheiden sich bald gelbe Krystalle $a b$, die durch Umkrystallisiren aus Weingeist leicht rein erhalten werden können. Der reine Körper bildet weisse Octaëder, die bei $237^{\circ}-238^{\circ}$ schmelzen und in wässrigem Natriumcarbonat und Natriumhydrat unlöslich sind. Die Analysen dieses Condensationsproduktes zeigten, dass auch in diesem Fall das Verhältniss von zwei Molekülen $\mathrm{C}_{12} \mathrm{H}_{12} \mathrm{O}_{2}$ zu einem Molekül des einwirkenden Reagens zur Wirkung kam, da die bei den Verbrennungen erhaltenen Zahlen sehr gut mit der Zusammensetzung $\mathrm{C}_{24} \mathrm{H}_{22} \mathrm{O}_{2}=\mathrm{NC}_{6} \mathrm{H}_{5}$ übereinstimmten:

$$
2 \mathrm{C}_{12} \mathrm{H}_{12} \mathrm{O}_{2}+\mathrm{C}_{3} \mathrm{H}_{5} \mathrm{NH}_{2}=\mathrm{C}_{24} \mathrm{H}_{28} \mathrm{O}_{2}=\mathrm{NC}_{8} \mathrm{H}_{5}+2 \mathrm{H}_{2} \mathrm{O} \text {. }
$$

Es ist uns nicht gelungen, durch Behandlung von $\mathrm{C}_{12} \mathrm{H}_{12} \mathrm{O}_{2}$ mit Alkohol und Salzsäure einen Säureäther desselben zu gewinnen. Die Verbindung $\mathrm{C}_{12} \mathrm{H}_{12} \mathrm{O}_{2}$ besitzt die empirische Zusammensetzung des Cinnamylacetons, und da die Bildung dieses Ketons bei den beschriebenen Reactionen wohl möglich war, haben wir es aus dem von Fischer und Kuzel ${ }^{1}$ ) dargestellten Cinnamylacetessigäther bereitet. $\mathbf{Z u}$ diesem $\mathbf{Z}_{\text {weck }}$ wurde der Aether mehrere Stunden mit Wasser gekocht und die Lösung alsdann mit Aether extrahirt. Man gewinnt ein halbflüssiges Produkt, welches nach der Reinigung Krystall-

1) Ber. 1883, 166. 
Natriumacetessig- $u$. Natriummalonsäureäthern etc. 393

blätter bildet, die bei $84^{\circ}$ schmelzen, und durch eine Analyse als das erwünschte Keton sich erwies. Der Körper ist unlöslich in wässrigem Kalihydrat und hat ganz andere Eigenschaften als unsere isomere Substanz.

Die Bildung von $\mathrm{C}_{12} \mathrm{H}_{12} \mathrm{O}_{2}$ aus $\mathrm{C}_{15} \mathrm{H}_{18} \mathrm{O}_{4}$ durch Alkalien repräsentirt nicht das letzte Stadium der Einwirkung, da, wenn man $\mathrm{C}_{12} \mathrm{H}_{12} \mathrm{O}_{2}$ längere Zeit mit wässrigen Alkalien erbitzt, eine weitere Zersetzung eintritt. Man erhält gefärbte Krystalle, die durch Krystallisation aus Ligroïn gereinigt werden können und die Zusammensetzung $\mathrm{C}_{12} \mathrm{H}_{14} \mathrm{O}_{3}$ besitzen. Diese Verbindung ist daher aus $\mathrm{C}_{12} \mathrm{H}_{12} \mathrm{O}_{2}$ durch Aufnahme von Wasser entstanden:

$$
\mathrm{C}_{12} \mathrm{H}_{12} \mathrm{O}_{2}+\mathrm{H}_{2}=\mathrm{C}_{13} \mathrm{H}_{14} \mathrm{O}_{3} \text {. }
$$

Sie bildet aus Ligroïn krystallisirt concentrische Gruppen von Nadeln, aus Wasser und Schwefelkohlenstoff grosse, harte Prismen, die bei $83^{\circ}-84^{\circ}$ schmelzen. Sie verhält sich wie eine Säure, indem sie in wässrigem Natriumcarbonat unter Kohlendioxydentwicklung sich auflöst. Mit verdünntem Kaliumpermanganat wird die wässrige Lösung nicht sogleich entfärbt, sie giebt demnach nicht die Reaction einer ungesättigten Säure. Mit $J_{0 d}$ in alkalischer Lösung entsteht Jodoform. Versetzt man eine neutrale Lösung der Verbindung in Ammoniak mit Silbernitratlösung, so fällt sogleich das Silberderivat als weisser Niederschlag von der Zusammensetzung

$$
\mathrm{C}_{12} \mathrm{H}_{13} \mathrm{O}_{3} \mathrm{Ag},{ }_{1} / \mathrm{H}_{2} \mathrm{O} \text {. }
$$

Der Körper $\mathrm{C}_{12} \mathrm{H}_{14} \mathrm{O}_{3}$ enthält ebenfalls ein Carbonyl, da er mit Hydroxylamin ein Oxim liefert. Man erhält es als eine amorphe Verbindung von der Zusammensetzung

$$
\mathrm{C}_{12} \mathrm{H}_{14} \mathrm{O}_{2} \mathrm{~N}(\mathrm{OH}) \text {. }
$$

Es löst sich leicht in Alkobol und Aether, sehr schwer in Ligroin und Benzol, und schmilzt unter Zersetzung bei $93^{\circ}-94^{\circ}$.

Von verdünnter Kaliumpermanganatlösung wird $\mathrm{C}_{12} \mathrm{H}_{14} \mathrm{O}_{3}$ leicht oxydirt, es gelang uns aber nur die Benzoësäure und das Silbersalz einer mit Wasserdampf flüchtigen Säure mit einem Silbergehalt von $58,8 \%$ bei dieser Oxydation zu gewinnen. Dieselbe Substanz wird ebenfalls von Phosphorpentachlorid leicht angegriffen unter Entstehung von Zimmtsäure.

Behandelt man $\mathrm{C}_{12} \mathrm{H}_{14} \mathrm{O}_{3}$ in alkoholischer Lösung mit Natriumamalgam, so tritt Reduction ein unter Bildung eines dick- 
flüssigen Oels, welches bei $271^{\circ}-273^{\circ}$ unter $17 \mathrm{~mm}$ Druck unzersetzt destillirt. Dieses Oel ist fast unlöslich in wässriger Natriumcarbonatlösung, aber in der Hitze geht es in Lösung und wird erst durch Zusatz einer Mineralsäure daraus gefällt. Das einmal gelöste und gefällte Produkt zeigt nun das gleiche Verhalten gegen Natriumcarbonatlösung, eine Eigenschaft, welche die Verbindung als ein Lacton charakterisirt. Das Lacton hat die Zusammensetzung $\mathrm{C}_{12} \mathrm{H}_{14} \mathrm{O}_{2}$ :

$$
\mathrm{C}_{12} \mathrm{H}_{14} \mathrm{O}_{3}+\mathrm{H}_{2}=\mathrm{C}_{12} \mathrm{H}_{14} \mathrm{O}_{2}+\mathrm{H}_{2} \mathrm{O} \text {. }
$$

Die Bildung dieses Lactons beweist ferner, dass $\mathrm{C}_{12} \mathrm{H}_{14} \mathrm{O}_{3}$ eine einbasische Carbonylsäure ist, eine Ansicht, die mit den sonstigen Eigenschaften derselben übereinstimmt.

Im Anfang dieser Untersuchung schien es von Interesse, die Synthese einer Verbindung von der Zusammensetzung $\mathrm{C}_{12} \mathrm{H}_{14} \mathrm{O}_{3}$ aus $\beta$-Bromphenylhydrozimmtsäureäther und Natriumacetessigäther zu versuchen, aber alle darauf hinzielende Versuche sind erfolglos geblieben, obwohl wir unter verschiedenen Bedingungen, sogar in ätherischer Lösung den Versuch angestellt haben. Der Natriumacetessigäther wirkte stets wie ein Alkali, unter Bildung von Zimmt- und Acetessigäthern und Bromnatrium.

Wie oben erwähnt, bleibt trotz dieser Untersuchung die Constitution von den Additions- und zuerst entstehenden Condensationsprodukten der Einwirkung von Natriumacetessigäther auf Zimmtsäureäther noch unaufgeklärt. Die früher vermuthete Constitution, wonach $\mathrm{C}_{18} \mathrm{H}_{16} \mathrm{O}_{4}$ von Tetramethylen sich ableiten solle, musste, abgesehen von dem Bedenken gegen die Existenz der Tetramethylengruppe, nach unseren gelegentlichen Versuchen aufgegeben werden. ${ }^{1}$ ) Es lässt sich nicht verkennen, dass die Bildung des Additionsproduktes ron der Zusammensetzung $\mathrm{C}_{6} \mathrm{H}_{5}-\mathrm{CH}-\mathrm{CH}-\mathrm{COOC}_{2} \mathrm{H}_{5} 2 \mathrm{CH}_{3}-\mathrm{CONa}-\mathrm{CH}-\mathrm{COOC}_{2} \mathrm{H}_{5}$ auf einen weit complicirteren Vorgang beim Natriumacetessigäther als beim Natriummalonsäureäther in ihrem Verhalten gegen Zimmtsäure hindeutet; und die Bildung von zwei Oximen aus $\mathrm{C}_{12} \mathrm{H}_{12} \mathrm{O}_{2}$ macht es wahrscheinlich, dass die Formel dieses Körpers zu verdoppeln ist. Es scheint dies auch aus dem

1) Dies. Journ. [2] 37, 499. 
Natrinmacetessig- u. Natriummalonsäureäthern etc. 395

Verbalten dieses Körpers gegen Anilin, wobei die Verbindung $\mathrm{C}_{24} \mathrm{H}_{22} \mathrm{O}_{2} \mathrm{NC}_{6} \mathrm{H}_{5}$ entsteht, hervorzugehen. Die Entstehung des Anilinderivats erinnert an das Verhalten des $\delta$-Diketones gegen organische Basen. Allein unser Produkt wird leicht durch Salzsäure in $\mathrm{C}_{12} \mathrm{H}_{12} \mathrm{O}_{2}$ und Anilin zersetzt, während, wenn es ein Pyrrolderivat wäre, man eine grössere Beständigkeit gegen Säuren erwarten müsste. Während die Constitutionen von $\mathrm{C}_{15} \mathrm{H}_{10} \mathrm{O}_{4}$ und $\mathrm{C}_{12} \mathrm{H}_{12} \mathrm{O}_{2}$ noch unaufgeklärt sind, berechtigten unsere Versuche über das weitere Zersetzungsprodukt $\mathrm{C}_{12} \mathrm{H}_{14} \mathrm{O}_{3}$ zu der Annahme, dass es eine einbasische Carbonylsäure und zwar von der Constitution

$$
\begin{gathered}
\mathrm{C}_{6} \mathrm{H}_{5}-\mathrm{CH}-\mathrm{CH}_{2}-\mathrm{COOH} \\
\mathrm{CH}_{2}-\mathrm{CO}-\mathrm{CH}_{3}
\end{gathered}
$$

ist, welche durch Reduction in $\delta$-Lakton<smiles>CC1CC(c2ccccc2)CC(=O)O1</smiles>

übergeht.

Es ist jedenfalls der auffallende Unterschied in dem Verhalten von Natriumacetessig- und von Natriummalonsäureäthern gegen Zimmtsäureäther nicht ohne Interesse. ${ }^{1}$ )

1) Seit längerer Zeit an der Fortsetzung meiner Arbeiten verhindert, habe ich erst vor kurzer Zeit meine Versuche über die Addition von Natriumderivaten zu ungesättigten Aethern wieder aufnehmen können. Mit Dr. O. Schulthess habe ich Versuche schon angestellt über das Verhalten von Akryl- und Fumarsäureäthern gegen Natriumacetessigäther. Die erste Reaction war von besonderem Interesse, da Wislicenus die Synthese des Acetglutarsäureäthers aus $\beta$-Brompropionsäureäther und Natriumacetessigäther glückte, und es könnte vielleicht aus dieser Reaction Aufschluss über die Wirkungsweise des Natriumacetessigäthers gewonnen werden. Wir înden, dass auch in diesem Fall die Addition sehr leicht stattfindet, aber das Additionsprodukt ist keineswegs identisch mit dem Wislicenus'schen Körper. Um zu zeigen, dass die Natriummalon\&äureätheraddition besonders geeignet ist zur Synthese von mehrbasischen Fettsäuren, haben wir das Verhalten derselben gegen Allylmalon-, Akryl-, Fumar-, Citra- und Itakonsäureäther untersucht. Mit Fumarsäureäther erhielten wir den bei $202^{\circ}-203^{\circ}(16 \mathrm{Mm}$. Druck) unzersetzt siedenden Aether, welcher bei der Verseifung mit concentrirter Kalilauge Tricarballylsäure liefert. Wir möchten uns das Studium des Verhaltens von Natriumacetessigäther gegen ungesättigte Säureäther, sowie von Natriummalonsäureäther gegen den oben erwähnten Aether auf einige Zeit vorbehalten.

A. M. 